\title{
Cardiac Resynchronization Treatment Upgrade is An Alternative Treatment Option for Resistant Ventricular Tachycardia After Double Valve Replacement
}

\author{
(1) Cihan İlyas Sevgican1, (1) Gökay Nar1, ㄴ Samet Yılmaz1, (1) Murat Biteker2
}

\footnotetext{
${ }^{1}$ Pamukkale University Faculty of Medicine, Department of Cardiology, Denizli, Turkey

${ }^{2}$ Muğla Sitkı Koçman University Faculty of Medicine, Department of Cardiology, Muğla, Turkey
}

\begin{abstract}
Ventricular tachycardia (VT) episodes may be fatal in patients with low ejection fraction. Current guidelines recommend implantable cardioverter defibrillator implantation after optimal medical treatment for these patients. Catheter ablation therapies should be considered in recurrent VT episodes. However, treatment options are limited in patients who cannot undergo catheter ablation.
\end{abstract}

\section{Introduction}

Ventricular tachycardia (VT) episodes may result hemodynamic collapse and death in patients with low ejection fraction heart failure. In these patients, implantable cardioverter defibrillator (ICD) implantation is a treatment option that reduces mortality ${ }^{(1)}$. Invasive catheter ablation
Hereby, we present a patient who had low ejection fraction, aortic and mitral valve replacement history with recurrent episodes of VT, and was not able to be planned invasive catheter ablation.

Keywords: Mechanical heart valve, ventricular tachycardia, cardiac resynchronization therapy

is considerable in patients with recurrent VT episodes with heart failure ${ }^{(2)}$. Factors such as hemodynamic instability, urgent procedure planning, advanced age, fragility and ischemic heart disease increase complication rates of ablation procedures. Access and ablate of substrates within ventricle may not always be possible due to

Address for Correspondence: Gökay Nar, Pamukkale University Faculty of Medicine, Department of Cardiology, Denizli, Turkey e-mail: gokay_nar@yahoo.com ORCID: orcid.org/0000-0001-6159-7785

Received: 24.12.2019 Accepted: 09.04.2020

Cite this article as: Sevgican CI, Nar G, Y1lmaz S, Biteker M. Cardiac Resynchronization Treatment Upgrade is An Alternative Treatment Option for Resistant VT After Double Valve Replacement. EJCM 2020;8(2):99-102.

DOI: 10.32596/ejcm.galenos.2019.12.064

${ }^{\circ}$ Copyright 2020 by Heart and Health Foundation of Turkey (TÜSAV) / E Journal of Cardiovascular Medicine published by Galenos Publishing House. 
changed anatomy in patients with structural heart disease. We present a resistant VT patient with a history of double valve replacement and left ventricle inaccessible due to mechanical heart valves for catheter ablation.

\section{Case Report}

A 57-year-old male patient with a history of aortic and mitral mechanical valve replacement and coronary artery bypass surgery 15 years ago and ICD that had been implanted in 2015 was referred to our clinic for recurrent VT episodes and ICD shocks despite intravenously high dose amiodarone treatment (Figure 1). Arterial blood pressure was $90 / 60 \mathrm{mmHg}$, pulse rate was $60 / \mathrm{min}$, and respiratory rate was $14 / \mathrm{min}$. On physical examination, he did not have any signs of hypervolemia. The patient

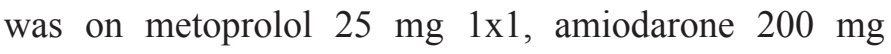
$2 \times 1$, spirinolactone $25 \mathrm{mg} \mathrm{1x}$, furosemide $40 \mathrm{mg} \mathrm{1x} 1$, and warfarin treatment. He could not receive optimal heart failure treatment due to hypotension. The patient was pacemaker dependent due to bradycardia (Figure 2A). Echocardiography revealed severely impaired left ventricular function $(25 \%)$ by Simpson's method, and left ventricular end-diastolic diameter was $6.8 \mathrm{~cm}$. Aortic and mitral mechanical prosthesis dysfunction was not

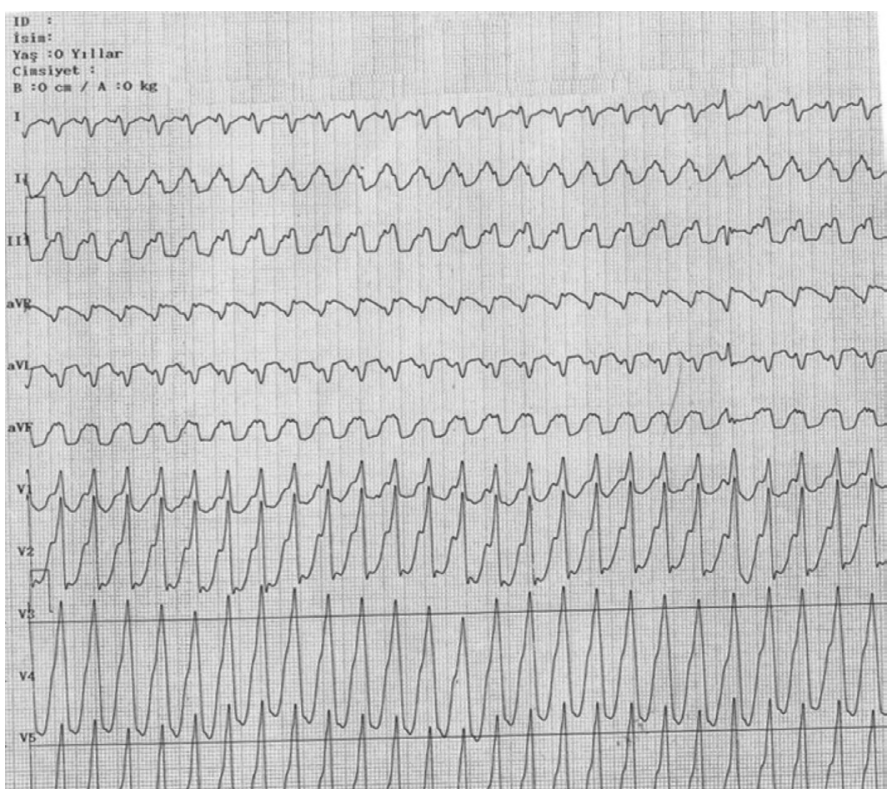

Figure 1. Left ventricle originated ventricular tachycardia detected. At admission, the patient's creatinine was 1.17 $\mathrm{mg} / \mathrm{dL}$, CRP was $0.125 \mathrm{mg} / \mathrm{dL}$, magnesium was $2.15 \mathrm{mg} /$ $\mathrm{dL}$, potassium was $4.7 \mathrm{mmol} / \mathrm{L}$, and hemoglobin was 14.3 $\mathrm{g} / \mathrm{dL}$ white blood cell (WBC) was $6.47 \mathrm{k} / \mathrm{uL}$. INR was in the optimal range. No extracardiac problem (electrolyte disturbances, hyperthyroidism, use of toxic drugs, etc.) which could cause recurrent VT episodes was found. Nine appropriate shock and multiple recurrent VT episodes were detected in the last 15 days.

Coronary angiography was performed, which showed coronary arteries without significant stenosis. Catheter ablation was considered due to the failure with antiarrhythmic treatment. However, the left ventricle could not be accessed by arterial and venous access due to mechanical aortic and mitral prostheses, so endocardial
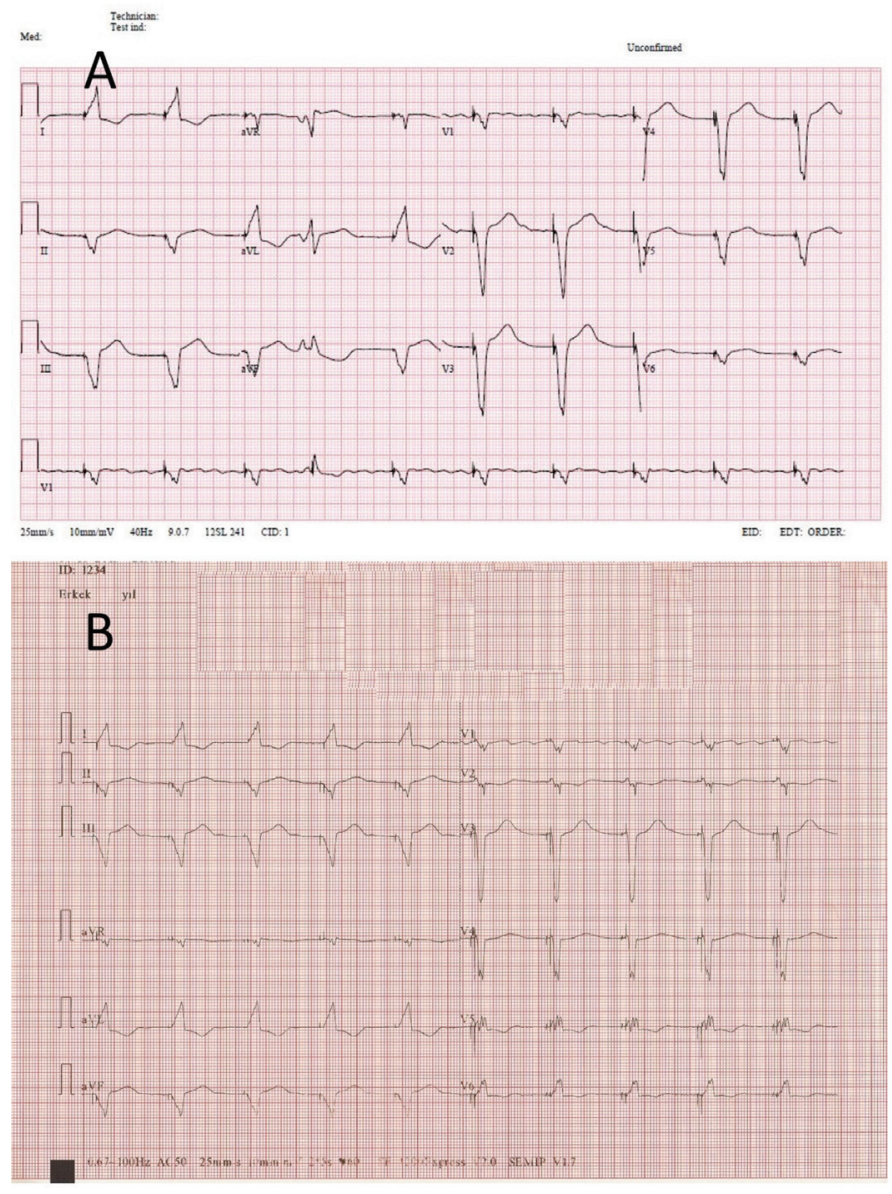

Figure 2. Pacemaker dependent heart rhythm due to bradycardia, baseline ECG $(A)$ of the patient, ECG recording of after cardiac resynchronisation treatment $(B)$ 
ablation was not performed. The patient refused epicardial catheter ablation due to procedural high risk. It was planned to optimize the treatment of the patient considering that frequent VT attacks might be caused by low flow rate due to heart failure. Since the ejection fraction of the patient was $25 \%$ and the QRS width was $>130 \mathrm{~ms}$ on his ECG, cardiac resynchronization therapy (CRT) upgrade was planned according to the current guidelines ${ }^{(1)}$. He had a history of ICD implantation and had an active right ventricular lead. The passive coronary sinus lead was advanced into the posterior branch of coronary sinus by subclavian vein puncture. The leads were connected to the Medtronic Protecta ${ }^{\mathrm{TM}}$ CRT-D battery and a biventricular pace rhythm was achieved (Figure 3).

After the CRT upgrade procedure, the patient's symptoms regressed and the QRS duration decreased from $153 \mathrm{msec}$ to $134 \mathrm{msec}$ (Figure 2B). No complex ventricular cardiac disorders were observed during in-hospital followup. The patient was discharged uneventfully. After three months, on control visit, ejection fraction increased from $25 \%$ to $29 \%$ (before and after treatment measured by the

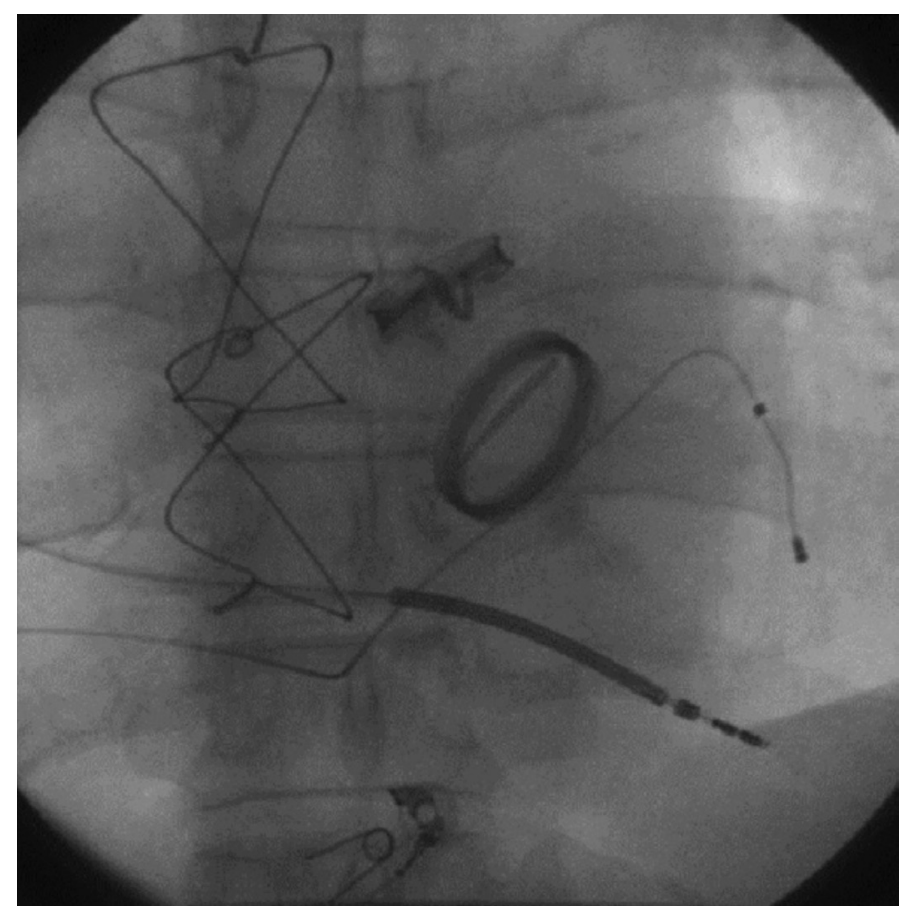

Figure 3. Fluoroscopy image of the patient after cardiac resynchronization treatment
Simpson's method with same echocardiography device) with provided cardiac synchronization. The patient had no symptoms of cardiac arrhythmia on control visit.

\section{Discussion}

The first arteries originating from the aorta are coronary arteries. Cardiac output is first used to supply the heart. In patients with ischemic heart disease, myocardial oxygen demand cannot be met and myocardial ischemia occurs when decreased cardiac output is added to the impaired vascular bed. The first effect of low cardiac output is myocardial ischemia and the second is impaired renal function, and the last effect is the inability to feed all of other organs. Increasing low cardiac output is important to meet myocardial oxygen demand and improve the heart's pumping function.

In ischemic heart disease, the electrical conduction on the myocardium is not homogeneous due to irregular scar areas. The electrical conduction on the damaged myocardial tissue between the scar areas cannot be as fast as the healthy myocardium. Due to all these electrical heterogeneity, micro and macro reentry circles may occur and VT episodes may be triggered. In these patients, maintaining myocardial synchronization is important for achieving electrical stability. In order to prevent this electrical heterogeneity, surroundings of the scar tissues are ablated and attempted to exclude heterogeneous electrical pathways ${ }^{(3,4)}$. ECG recordings were evaluated and VT was thought to be of left ventricular origin. Due to the history of double valve replacement, left ventricle were not reached and catheter ablation was not performed. For the purpose of avoiding re-entry circles that cannot be treated by catheter ablation, pacing with synchronized right and left ventricles is also an option ${ }^{(5)}$.

Sustained chronic mechanical stretch also shortens the myocytes-effective refractory periods and the mean action potential durations while prolonging activation times. These changes result in electromechanical remodeling in the chronic period. As a result of chronic heart failure, this 
condition serves as a cause for stretch-induced ventricular $\operatorname{arrhtymogenicity}^{(3)}$.

It is reported in the literature that the recurrence rate in the treatment of scar-related VT with catheter ablation in patients with ischemic heart disease can reach up to $50 \%$ in the first 6 months $^{(4,5)}$. Treatment options are limited in patients with recurrent VT episodes in which catheter ablation cannot be performed. Secondary causes (electrolyte disturbance, hyperthyroidism, infectious processes, anemia, hypoxia, etc.) should be treated first in patients with VT episodes, and then examined for myocardial ischemia. After exclusion of channelopathies and non-cardiac effects, treatment is planned according to the presence of structural heart disease, patient characteristics and presence of ischemic heart disease. In addition to these treatment options, studies on cardiac radiotherapy have been increasing over time in patients who did not receive successful treatments ${ }^{(6)}$. Long-term oral antiarrhythmic treatment in patients with or without structural heart disease also brings drug side effects.

In our patient, invasive catheter ablation was considered due to ischemic heart disease and recurrent VT attacks. Access to the left ventricle is impossible due to aortic and mitral mechanical prostheses. The procedure was not performed. Another option would be to reach the left ventricle with the transapical approach for invasive ablation, but the procedure was considered to be highrisk due to the patient's general condition and was not performed. Such successfully cases were reported in literature ${ }^{(7)}$.

CRT implantation may be considered as a treatment option in ventricular arrhythmias triggered by low output and bradycardia because of provided adequate heart rate and improved cardiac output. Multifocal ventricular arrhythmias are not suitable for catheter ablation. Ventricular arrhythmias that can be treated with overdrive pacing and patients with advanced heart failure, who have multifocal ventricular arrhythmias, can benefit from CRT treatment.
In conclusion, CRT implantation may be considered among the treatment options in recurrent VT patients who cannot perform ablation procedures to correct low cardiac output and provide cardiac synchronization.

\section{Ethics}

Informed Consent: Informed consent of the patient was obtained.

Peer-review: Internally and externally peer-reviewed.

\section{Authorship Contributions}

Surgical and Medical Practices: G.N., Concept: M.B., Design: S.Y., Data Collection or Processing: C.İ.S., Analysis or Interpretation: S.Y., Literature Search: G.N., Writing: C.İ.S.

Conflict of Interest: The authors declared no conflict of interest.

Financial Disclosure: No financial support for this article.

\section{References}

1. Ponikowski P, Voors AA, Anker SD, Bueno H, Cleland JGF, Coats AJ S, et al. 2016 ESC Guidelines for the diagnosis and treatment of acute and chronic heart failure. Eur. Heart J 2016;37:2129-200.

2. Al-Khatib SM, Stevenson WG, Ackerman MJ, Bryant WJ, Callans DJ, Curtis AB, et al. AHA/ACC/HRS Guideline for Management of Patients With Ventricular Arrhythmias and the Prevention of Sudden Cardiac Death: A Report of the American College of Cardiology/American Heart Association Task Force on Clinical Practice Guidelines and the Heart Rhythm Society J Am Coll Cardiol 2018;138:e272-e391.

3. Alvarez CK, Cronin E, Baker WL, Kluger J. Heart failure as a substrate and trigger for ventricular tachycardia. J Interv Card Electrophysiol 2019;56:229-47

4. Spartalis M, Spartalis E, Tzatzaki E, Tsilimigras DI, Moris D, Kontogiannis C, et al. Novel approaches for the treatment of ventricular tachycardia. (2018); World J. Cardiol 2018;10:52-9.

5. Peichl P, Wichterle D, Pavlu L, Cihak R, Aldhoon B, Kautzner J. Complications of catheter ablation of ventricular tachycardia: a singlecenter experience. Circ. Arrhythm. Electrophysiol 2014;7:684-90.

6. Zei PC, Mak R. Noninvasive Stereotactic Radioablation for Ventricular Tachycardia. Circulation 2019;139:322-4.

7. Vurgun VK, Altin AT, Kilickap M, Candemir B, Akyurek O. Percutaneous transapical approach and transcatheter closure for ventricular tachycardia ablation. Pacing Clin. Electrophysiol. 2018;41:334-7. 